\title{
Numerical methods for solving inverse problems of fluid filtration to a horizontal well
}

\author{
Elena Badertdinova ${ }^{1, *}$, and Rustem Khairullin ${ }^{1}$ \\ ${ }^{1}$ Kazan National Research Technological University, 420015, K. Marks str. 68, Kazan, Russia
}

\begin{abstract}
In this paper, a three-dimensional mathematical model of thermohydrodynamic processes occurring in an oil reservoir exposed by a horizontal well after its launch is constructed. Based on the proposed model and the theory of ill-posed problems, a numerical method is proposed for solving the inverse coefficient problem for determining the filtration properties of an oil reservoir. As the initial information, temperature change curves taken simultaneously by several deep autonomous measuring instruments installed in various sections of the horizontal well bore are used. According to the proposed method, the heterogeneity of the reservoir is evaluated along the horizontal part of the well bore.
\end{abstract}

\section{Introduction}

In the last decade, in connection with the creation of sensitive in-depth measuring equipment, new technologies for conducting a field experiment and methods for interpreting the results of a field experiment have appeared. Problems associated with the interpretation of geological and field information lead to mathematical problems that are incorrect in the sense of Adamard. The numerical solution of such problems requires the development of special methods. One of the effective approaches to solving such problems is proposed by A.N. Tikhonov principle of narrowing the class of possible solutions, taking into account a priori information about the desired object. A distinctive feature of the inverse problems of oil and gas hydromechanics related to the study of mathematical models of real filtration processes in oil reservoirs is that the nature of the additional information is determined by the capabilities of a field experiment. In $[1,2]$, a method is proposed for determining the filtration properties of a reservoir opened by a horizontal well by the stationary distribution of temperature and pressure along the wellbore. In [3], the problem of determining the filtration properties of a stratified reservoir uncovered by a vertical well by measuring bottomhole pressures and temperatures in each layer (multisensor technology) is considered. In [4], the problem of determining the filtration properties of an oil reservoir exposed by a horizontal well when the reservoir consists of several isolated layers along the wellbore with various filtration properties is considered. In this paper, we propose a numerical method for solving the three-dimensional inverse coefficient problem for determining the filtration properties of an oil reservoir.

\footnotetext{
* Corresponding author: badertdinova@yandex.ru
} 


\section{Statement of the direct problem}

Measurements of temperature and pressure in a horizontal wellbore by deep-well equipment provide complete information about thermohydrodynamic processes occurring in the reservoir. The change in temperature in the horizontal wellbore is an integral indicator of heat and mass transfer processes occurring both in the well itself and in the reservoir. Full stabilization of the hydrodynamic or thermohydrodynamic state of the reservoir system is possible only after equilibrium of both pressure and temperature is established. It should be noted that the pressure field is restored much faster than the temperature field. The case of a fast process of redistribution of the pressure field can be observed on the model of a rigid porous system saturated with an incompressible fluid. In such a system, the stationary pressure distribution is achieved almost instantly and, at the same time, the rate of temperature redistribution in the reservoir remains real [5].

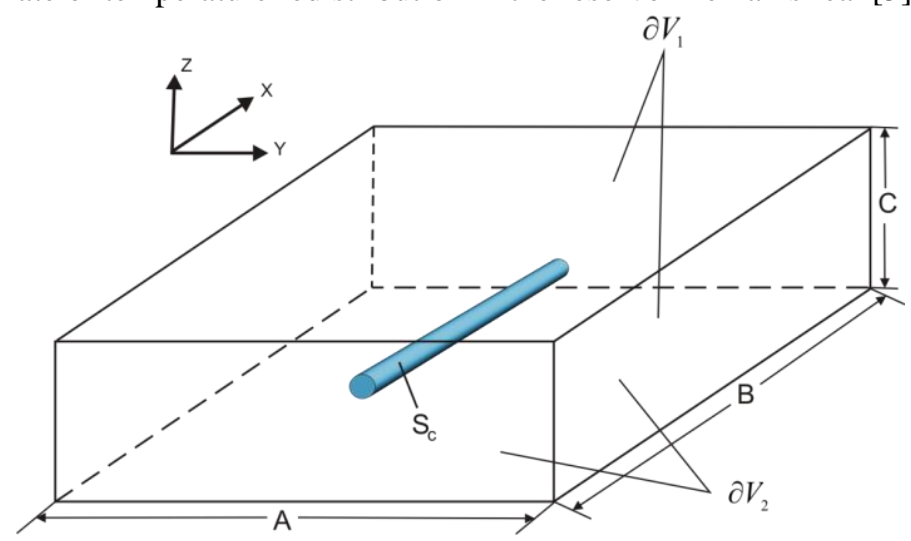

Fig. 1. The reservoir model

It is assumed that the horizontal wellbore is parallel to the roof and the bottom of the reservoir, the process of pressure distribution in the formation is established (Fig. 1). In this case, the process of nonisothermal filtration to a horizontal well is described by the following equations:

$$
\begin{aligned}
& \quad \operatorname{div}(k / \mu \operatorname{grad} p)=0, \\
& C_{P} \cdot \partial T / \partial t=\operatorname{div}(\lambda \operatorname{grad} T)+\rho C_{g} \cdot k / \mu \cdot \operatorname{grad} p(\operatorname{grad} T+\operatorname{ggrad} p)=0, \quad 0<t \leq t \operatorname{texp}
\end{aligned}
$$

with the following initial:

and boundary conditions:

$$
p(x, y, z, 0)=p O(x, y, z), T(x, y, z, 0)=T O(x, y, z)
$$

$$
\begin{aligned}
& \partial p /\left.\partial z\right|_{z=0}=0, \partial p /\left.\partial z\right|_{z=C}=0, \partial T /\left.\partial z\right|_{z=0}=0, \quad \partial T /\left.\partial z\right|_{z=C}=0, \\
& \left.p\right|_{x=0}=p k,\left.p\right|_{x=A}=p k,\left.p\right|_{y=0}=p k,\left.p\right|_{y=B}=p k, \\
& \left.T\right|_{x=0}=T k,\left.T\right|_{x=A}=T k,\left.T\right|_{y=0}=T k,\left.T\right|_{y=B}=T k, \\
& Q=\int_{S} k / \mu \cdot \partial p / \partial n d s, \\
& C_{P} \cdot \partial T / \partial t-\rho C_{g} \cdot k /\left.\mu \cdot \operatorname{gradp}(\operatorname{grad} T+\operatorname{ggrad} p)\right|_{S}=0, \quad 0<t \leq \operatorname{texp}
\end{aligned}
$$

where $k(\mathrm{x}, \mathrm{y}, \mathrm{z})$ is the permeability, $\mu$ is the viscosity, $q$ is the well flow rate, $p=p(\mathrm{x}, \mathrm{y}, \mathrm{z})$, $T=T(\mathrm{x}, \mathrm{y}, \mathrm{z}, \mathrm{t})$ is the pressure and temperature of the formation, $p_{\mathrm{k}}$ is the reservoir pressure, $T_{\mathrm{k}}$ is the reservoir temperature, $r_{\mathrm{c}}$ is the radius of the wellbore, $\varepsilon$ is the Joule-Thomson coefficient. Fluid flow per unit surface area of a horizontal wellbore is calculated from the assumption that surface pressure $\mathrm{S}$ is constant. 


\section{Analysis of the solution of the direct problem}

System (1)-(8) is solved numerically by the finite difference method [6]. The reservoir, which is a doubly connected filtration region, is covered by an uneven finite-difference grid. In the region of greatest gradients (near-bottom zone), the mesh is thickened using the logarithmic transformation of coordinates along the OY, OZ axes. Along the horizontal trunk along the $\mathrm{OX}$ axis, local mesh refinement is performed. For the finite-difference approximation of the convective term in equation (2), the "upstream" procedure is used [6].

Next, an analysis is made of the influence of reservoir parameters on the temperature curves measured in the wellbore.

A model heterogeneous oil reservoir, which is being developed by a horizontal well, is considered. A horizontal well is put into operation with constant fluid withdrawal from the reservoir. The calculations were carried out with the following parameters: $C_{P}=1.4810^{6}$ $J /\left(m^{3} \mathrm{~K}\right), C_{g}=1929 \mathrm{~J} /(\mathrm{kgK}), \mathrm{T}_{\mathrm{k}}=300 \mathrm{~K}, \mathrm{p}_{\mathrm{k}}=10 \mathrm{MPa}, \mu=25 \mathrm{MPa} \mathrm{s}, \rho=800 \mathrm{~kg} / \mathrm{m}^{3}, \varepsilon=0.4$ $K / M P a, A=100 \mathrm{~m}, \mathrm{~B}=100 \mathrm{~m}, \mathrm{C}=10 \mathrm{~m}, L=50 \mathrm{~m}, r_{\mathrm{c}}=0.1 \mathrm{~m}, q=10 \mathrm{~m}^{3} /$ day. It is assumed that the permeability of the reservoir is a piecewise constant function. In each zone of uniformity of the reservoir, a downhole tool is located (Fig. 2). The following arrangement of formation homogeneity zones is considered: $k_{1}<k_{2}, k_{1}=0.05 \mu \mathrm{m}^{2}, k^{2}=0.1 \mu \mathrm{m}^{2}$.

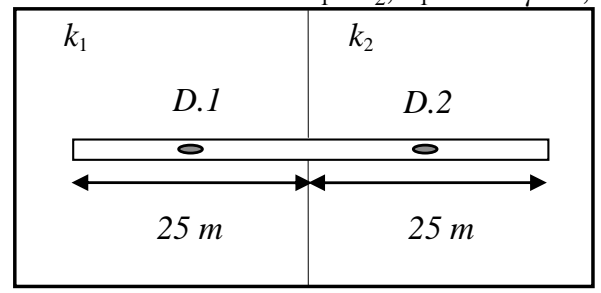

Fig. 2. Device locations in a heterogeneous formation

Next, we study the influence of the permeability coefficients of the homogeneity zones $k_{1}$, $k_{2}$ and Joule-Thomson $\varepsilon$ on the curves of temperature change. The curves of temperature changes measured by devices 1, 2 (Fig. 2) in the homogeneity zones are shown in Fig. 3.

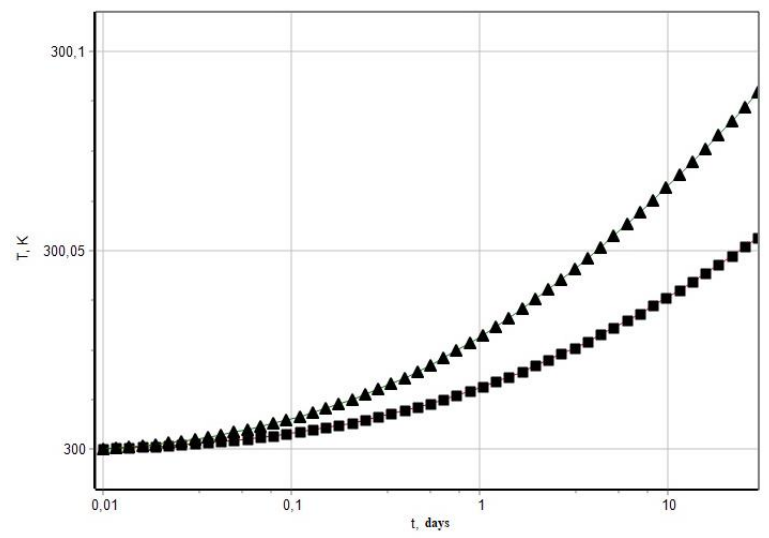

Fig. 3. Curves of temperature change. $\mathbf{a}$ - device 1, $\mathbf{\Delta}$ - device 2 .

Fig. 4-5 show the results of the calculations. From the calculation results it follows that the permeability and Joule-Thompson coefficients significantly affect the process of temperature change (Fig. 4). 


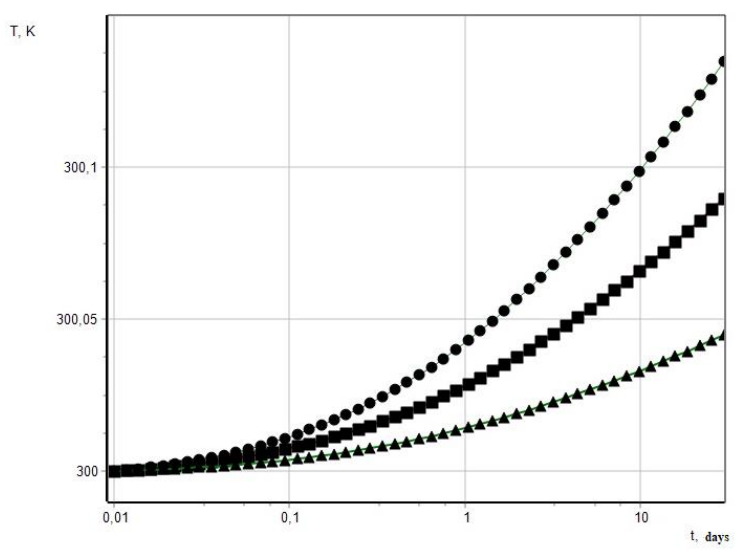

Fig. 4. Curves of temperature change (influence of the Joule-Thomson coefficient). $\boldsymbol{\Delta}-\varepsilon=0.2$ $\mathrm{K} / \mathrm{MPa},-\varepsilon=0.4 \mathrm{~K} / \mathrm{MPa}, \bullet-\varepsilon=0.6 \mathrm{~K} / \mathrm{MPa}$

The results of a computational experiment showed that the curves of temperature changes at the bottom of the well are little sensitive to changes in the coefficients of heat capacity and thermal conductivity (Fig. 5).

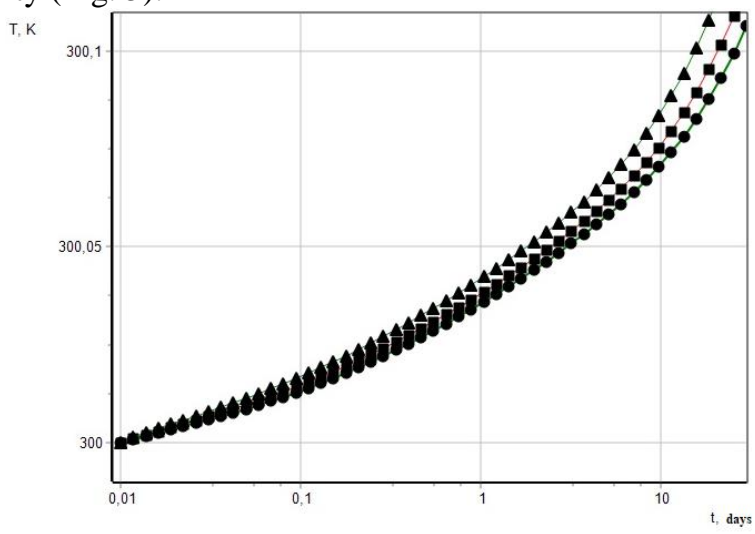

Fig. 5. Curves of temperature change. $\boldsymbol{\Delta}-\mathrm{Cp}=1.5 .106 \mathrm{~J} /(\mathrm{kg} \mathrm{K}), \boldsymbol{-}-\mathrm{Cp}=2.016 .106 \mathrm{~J} /(\mathrm{kg} \mathrm{K}), \bullet-$ $\mathrm{Cp}=2.5 .106 \mathrm{~J} /(\mathrm{kg} \mathrm{K})$

Based on the sensitivity analysis, it can be concluded that the temperature change curves measured by depth instruments can be used as initial information for estimating the permeability coefficient of an oil reservoir.

\section{Statement and solution of the inverse coefficient problem}

Based on the proposed mathematical model, a computational algorithm for interpreting the results of thermohydrodynamic studies of horizontal wells is proposed. Plots for placement of deep measuring equipment are determined on the basis of geophysical studies of the well. The work [7] describes the technology for conducting thermohydrodynamic studies of a horizontal well using several deep autonomous devices.

Let the temperature curves $\phi_{j}(\mathrm{t}), j=1,2, \ldots, N, 0<t \leq t_{\text {exp }}$, be taken at the locations of deepseated measuring instruments in the horizontal well bore with coordinates $\gamma_{j}, j=1,2, \ldots, N$.

The inverse coefficient problem is formulated as follows: 
determine the permeability coefficient $k(\mathrm{x}, \mathrm{y}, \mathrm{z})$, when thermohydrodynamic processes in the oil reservoir are described by equations (1) - (8). As the initial information, the measured temperature values are used by deep autonomous devices.

An estimate of the permeability coefficient is sought in the class of piecewise constant functions $k(\mathrm{x}, \mathrm{y}, \mathrm{z})=k_{n},(\mathrm{x}, \mathrm{y}, \mathrm{z}) \in V_{n}, \mathrm{U}_{\mathrm{n}=1}{ }^{\mathrm{N}} V_{n}=V$, где $V_{n}, n==1,2, \ldots, N$ - areas of homogeneity (Fig. 2).

A numerical solution of the inverse coefficient problem (1) - (9) is sought from minimizing the standard deviation between the observed and calculated values:

$$
F(a)=\sum_{n=1}^{N} \int_{0}^{t \exp }\left(T\left(\gamma_{n}, t\right)-\phi_{n}(t)\right)^{2} d t
$$

where $\phi_{n}(\mathrm{t})$ are the observed temperature values, $T\left(\gamma_{n}, t\right)$ are the calculated temperature values obtained from the numerical solution of equations (1) - (8), $\alpha=\left(k_{1}, k_{1}, \ldots, k_{n}\right)$ is the desired parameter, $0<m_{n} \leq k_{n} \leq M_{n}, m_{n}, M_{n}=$ const.

The computational algorithm for minimizing the standard deviation (9) is based on the Levenberg-Marquardt method:

$$
\alpha^{l+1}=\alpha^{l}-\left(H^{l}+v E\right)^{-1} \nabla F
$$

where $H^{l}$ is the approximate matrix of second derivatives, $H^{l}=A^{T} A,\{A\}=\left\{\partial \partial F_{i} / \partial \alpha_{j}\right\}$ is the sensitivity matrix, $v$ is the regularization parameter, $E$ is the identity matrix, $v F$ is the gradient of the residual functional $\mathrm{F}\left(\alpha^{l}\right)=\sum_{\mathrm{i}=1}{ }_{\tau} F_{\mathrm{i}}$.

$F_{\mathrm{i}}=\sum_{\mathrm{i}=1} \mathrm{~N}\left(T\left(\gamma_{n}, t_{i}\right)-\phi_{n}\left(t_{\mathrm{i}}\right)\right)^{2} \tau_{\mathrm{i}}, N_{\tau}$ is the number of temperature measurements, $l$ is the iteration number.

The criteria for stopping the iterative process are the fulfillment of at least one of the conditions: $\left|\mathrm{F}\left(\alpha^{l-1}\right)-\mathrm{F}\left(\alpha^{l}\right)\right|<\varepsilon_{1},\left|\alpha^{l+1}-\alpha^{l}\right|<\varepsilon_{2}$, где $\varepsilon_{1}$ и $\varepsilon_{2}$, where $\varepsilon 1$ and $\varepsilon 2$ are given positive numbers.

A model oil reservoir is considered with the data given in paragraph 3. Based on these data, model curves of temperature change are calculated, which are then used as initial information. In Figure 6 shows the results of a numerical experiment that shows the convergence of an iterative process.

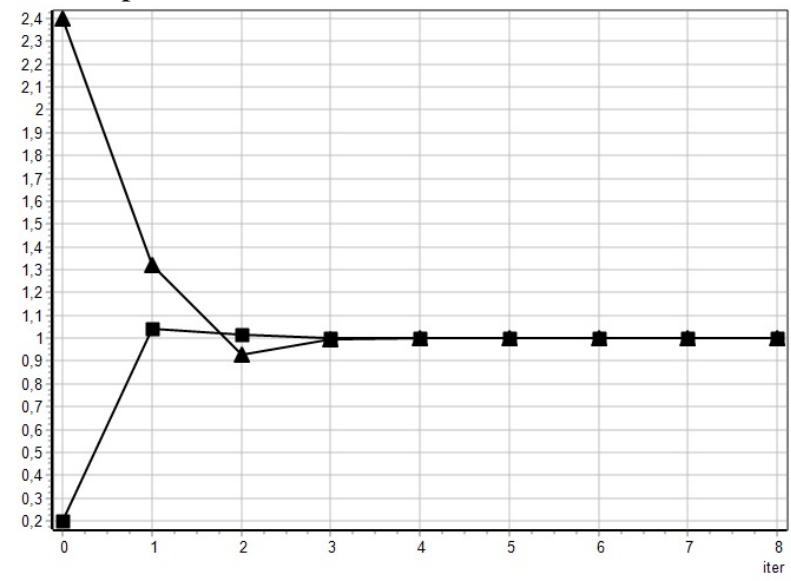

Fig. 6. Convergence of the iterative process.

To study the stability of the proposed algorithm, errors were randomly introduced into the model curves of temperature changes. The results of numerical experiments showed that the proposed computational algorithm is stable with respect to the error of the initial information and allows one to evaluate the filtration properties of the formation with sufficient accuracy for practice. 


\section{References}

1. K. Yoshika, D. Zhu, A.D. Hill, SPE 94097 (2005)

2. K. Yoshioka, D. Zhu, A. D. Hill, L. W. Lake, SPE Prod \& Oper, 24 (4), 510-521 (2009)

3. W. Sui, D. Zhu, A.D. Hill, C.A. Ehlig-Economides, SPE-116270 (2008)

4. M. Khairullin, M. Shamsiev, E. Baderddinova, A. Abdullin, Thermophysics of high temperatures, 50 (6), 830-834 (2012)

5. E. B. Chekalyuk Thermodynamics of the oil reservoir, Moscow, Nedra, (1965)

6. M. Kh. Khairullin, R. S. Khisamov, M. N. Shamsiev, R. G. Farkhullin Interpretation of the results of hydrodynamic studies of wells using regularization methods Moscow, Institute for Computer Research (2006)

7. A. A. Samarsky, Theory of difference schemes. Moscow, Nauka, (1983) 